TITLE:

\title{
The impact of complete lymph node dissection for lung cancer on the postoperative course.
}

\author{
AUTHOR(S): \\ Date, Hiroshi
}

\section{CITATION:}

Date, Hiroshi. The impact of complete lymph node dissection for lung cancer on the postoperative course.. Thoracic surgery clinics 2012, 22(2): 239-242

\section{ISSUE DATE:}

2012-05

URL:

http://hdl.handle.net/2433/155458

\section{RIGHT:}

(c) 2012 Elsevier Inc.; This is not the published version. Please cite only the published version.; この論文は出版社版でありません。引用の際に は出版社版をご確認ご利用ください。 
Thoracic Surgery Clinics

THE LYMPHATIC SYSTEM IN THORACIC ONCOLOGY

Review

\section{The impact of complete lymph node dissection for lung cancer on the postoperative course}

\section{Hiroshi Date, MD}

The Department of Thoracic Surgery, Kyoto University Graduate School of Medicine, Kyoto Japan

The author has nothing to disclose.

Key words: lung cancer, lymph node dissection, lymph node sampling, lymph node biopsy, survival, staging

Address for correspondence:

Hiroshi Date, MD

Professor and Chairman

Department of Thoracic Surgery

Kyoto University Graduate School of Medicine

54 Shogoin-Kawahara-cho, Sakyo-ku, Kyoto 606-8507, Japan

TEL: +81-75-751-4975, FAX: +81-75-751-4974

E-mail: hdate@kuhp.kyoto-u.ac.jp 


\section{SYNOPSIS}

- The role of lymph node dissection (LND) for non-small cell lung cancers remains controversial.

- LND adds little morbidity to a pulmonary resection for NSCLC although it requires additional operative time by $15-20 \mathrm{~min}$.

- Four prospective randomized trials have been performed to compare lymph node sampling (LNS) and LND; three trials showed no difference in survival and one trial showed survival benefit of LND.

- The largest prospective randomized trial ACSOG Z0030 clearly demonstrated that LND does not improve long-term survival in patients with early-stage (T1 or T2, N0 or non-hilar N1) NSCLC who have pathologically negative lymph nodes after rigorous systematic LNS.

- It is recommended that all patients with resectable NSCLC should undergo LND because the procedure provides the patients with the most accurate staging and the opportunity for effective adjuvant therapy. 


\section{INTRODUCTION}

It is well known that metastasis to hilar or mediastinal lymph node, the $\mathrm{N}$ factor, is one of the most important determinants of prognosis after lung cancer surgery (1-3). However, the role of lymphadenectomy in the staging and treatment of non-small cell lung cancer (NSCLC) remains controversial. Current surgical practice varies from visual inspection of the unopened mediastinum to systemic lymph node dissection. Systematic mediastinal lymph node dissection, as practiced by Japanese surgeons, is a much more extensive operation than that practiced by North American surgeons. Little and colleagues surveyed 729 hospitals to retrieve information on the patterns of surgical care provided to patients with NSCLC (4). The report included more than 11,000 patients with major pulmonary resections and showed that only $57.3 \%$ of patients had any mediastinal lymph nodes removed at the time of operation.

The purposes of hilar and mediastinal lymph node dissection are accurate staging and the improvement of survival though better local control. The accurate staging is very important since several large-scale randomized trials have shown that postoperative adjuvant chemotherapy can improve the survival after surgery for NSCLC $(5,6)$. There is no doubt that complete lymph node dissection is the most accurate way to stage a patient's disease and to determine which patients might benefit from adjuvant therapy. However, the extent of lymph node removal required and the impact of mediastinal lymph node removal on survival are still controversial.

In this chapter, the impact of lymph node dissection for NSCLC on the postoperative course, namely morbidity and survival, is reviewed and discussed.

\section{DEFFINITIONS}

Although nodal staging of NSCLC should be as accurate as possible, the surgical technique for lymph node assessment varies among countries and centers. It should be 
also noted that different terms are used to describe these surgical techniques. The definitions for various types of lymph node assessment were recently proposed by the council of the European Society for Thoracic Surgeons (7).

Lymph node biopsy

Only suspicious one or multiple lymph node(s) are biopsied to prove N1 or N2 disease. No systematic nodal dissection or biopsies are performed. The procedure is performed only to document lymph node metastasis when resection is not justified (Figure 1A).

\section{Lymph node sampling}

One or more lymph nodes are removed without any surrounding tissue, guided by preoperative or intraoperative findings that are thought to be representative (Figure 1B). Systematic lymph node sampling means that a predetermined sampling of the lymph node stations is performed.

Lymph node dissection

The mediastinal tissue containing lymph nodes is dissected and removed systematically within anatomical landmarks (Figure 1C). In other words, all of the lymph nodes in the targeted compartment must be completely removed, as a block, together with surrounding adipose tissue (8). In general, following lobe-specific lymph node dissection should be performed:

Right upper and middle lobe: 2R, 4R and 7;

Right lower lobe: 4R, 7, 8, and 9;

Left upper lobe: 5, 6 and 7;

Left lower lobe: 7, 8 and 9;

\section{COMPARISON LYMPH NODE SAMPLING AND LYMPH NODE DISSECTION}

\section{Mortality and Morbidity}

Some surgeons believe that pulmonary resection with lymph node sampling (LNS) is 
preferable for patients with NSCLC because lymph node dissection (LND) can be associated with significantly increased morbidity and most recurrence are distant rather than local. In contrast, the standard approach for NSCLC in Japan has been pulmonary resection with systematic LND believing it can achieve better local control with negligible LND related morbidity.

Reasons not to perform LND include prolonging the operation and causing more operative complications such as recurrent nerve injury, chylothorax, bronchial devascularization, increased lymphatic drainage and bleeding. Izbicki et al. reported that LND did not increase the postoperative mortality or re-thoracotomy rate in their prospective randomized trial (9), but the mean duration of operation increased by 20 min. Allen et al. reported 30-day operative data from 1,111 patients in a randomized trial comparing LNS and LND (10). Operative mortality was $2.0 \%$ for LNS and $0.8 \%$ for LND. Complication occurred in $38 \%$ of patients in each group. They also found an increase in operative time by $15 \mathrm{~min}$. These studies suggested that LND adds little morbidity to a pulmonary resection for NSCLC although it requires additional operative time by $15-20 \mathrm{~min}$.

\section{Survival}

So-called stage migration phenomenon between LNS and LND is an important factor that produces bias in retrospective studies. The best evidence is based on prospective randomized trials. Four prospective randomized trials to compare LNS and LND have been performed in Germany (9, 11), Japan (12), China (13) and USA (10, 14, 15). Inclusion criteria and methodologies are quite diverse among these studies, therefore, interpretation of each study has to be made carefully.

\section{(1) German study $(9,11)$}

Inclusion criteria: resectable NSCLC based on CT 
Number of patients: LNS $(\mathrm{n}=93), \operatorname{LND}(\mathrm{n}=76)$

Median follow-up: 47.5 months

Survival: No difference

This is the first prospective randomized trial comparing LNS and LND. Two hundred one patients were recruited for the study, but 32 patients were excluded from analysis due to various reasons. Somewhat LNS group included 17 patients more than LND group. About $60 \%$ of patients were node-negative pathologically in each group. There was no difference in survival between LNS and LND $(p=0.256)$. However, they found a borderline effect on survival $(\mathrm{p}=0.058)$ in patients in patients with $\mathrm{pN} 1$ or single level pN2. They concluded that LND does not influence survival for patients without overt lymph node involvement but might provide benefit for patients with limited lymph node metastases.

\section{(2) Japanese study (12)}

Inclusion criteria: peripheral NSCLC less than $2 \mathrm{~cm}$, cN0 based on CT

Number of patients: LNS $(\mathrm{n}=56), \operatorname{LND}(\mathrm{n}=59)$

Median follow-up: 65 months

Survival: 5-year survival LNS (84\%) $\fallingdotseq \mathrm{LND}(81 \%)$

This is a small but well designed study comparing LNS and LND for patients with clinical N0 small (less than $2 \mathrm{~cm}$ ) NSCLC. Pathological N2 was found in $14 \%$ and $12 \%$ of LNS group and LND group respectively. Survival was similar and no significant differences in the recurrence rate or survival was seen between the two groups. They concluded that clinically evaluated peripheral NSCLC smaller than $2 \mathrm{~cm}$ do not require LND.

(3) Chinese study (13) 
Inclusion criteria: resectable stage I-IIIA based on CT

Number of patients: LNS ( $\mathrm{n}=264), \mathrm{LND}(\mathrm{n}=268)$

Median follow-up: more than 5 years

Survival: median survival LND (59 months) > LNS (34 months)

This is a larger trial of 532 patients with clinical stage I-IIIA NSCLC. As compared to the previous study, only about $30 \%$ of enrolled patients had stage I disease and $70 \%$ of them had nodal involvement. Survival was significantly better in LND group than in LNS group. When the two groups were compared in subgroups according to pathological stage, LND group provided significantly better survival than LNS group in stage I and stage IIIA. This study has been the only randomized trial suggesting the survival benefit of LND.

(4) American study $(10,14,15)$

Inclusion criteria: N0 or non-hilar N1, T1 or T2

All negative lymph nodes after systematic LNS

Number of patients: LNS $(\mathrm{n}=498), \operatorname{LND}(\mathrm{n}=525)$

Median follow-up: 6.5 years

Survival: median survival LNS (8.5 years) $\fallingdotseq$ LND (8.1 years)

The American College of Surgeons Oncology Group (ASOSOG) has performed a randomized multi-institutional prospective trial designed to determine whether long-term survival is affected by LND versus LNS at the time of pulmonary resection for NSCLC (ACOSOG Z0030 trial). Of note, this study was not a simple comparison of LNS and LND, but designed to assess the efficacy of additional LND when systematic LNS was negative. Systematic LNS was performed at $2 \mathrm{R}, 4 \mathrm{R}, 7$, and $10 \mathrm{R}$ for right-sided tumors and 5, 6, 7, and 10L for left-sided tumors. Occult N2 disease was found in 4\% by the additional LND. Survival was similar between LNS and LND groups. There was no 
difference in local, regional, or distant recurrence between the two groups. They concluded that if systematic LNS is negative, LND does not improve survival in patients with early stage NSCLC.

Although these four studies were all prospective, the Chinese study was the only one which showed survival benefit of LND and other three studies showed no significant benefit. This difference may be derived from the different inclusion criteria used in each study. One can assume that LND may provide better local control and better survival than LNS only when there is positive lymph node dissected. In other words, when there is no positive lymph node dissected, LND would never be beneficial theoretically. Therefore, how many of the patients enrolled had positive lymph node would affect the outcome of these randomized trial comparing LNS and LND. The ratio of stage I (no lymph node involved) was about $60 \%$ in German study, $30 \%$ in Chinese study, and $80 \%$ in Japanese and American studies. Interestingly, Japanese and American studies (stage I, 80\%) showed no survival benefit of LND, German study (stage I, 60\%) showed borderline effect of LND on survival in patients with $\mathrm{pN} 1$ or single level $\mathrm{pN} 2$, and Chinese study (stage I, 30\%) showed significant benefit of LND. In a non-randomized trial, Keller and his colleagues reported the comparison of survival among patients with stage II-IIIA NSCLC who underwent either LNS or LND in the Eastern Cooperative Oncology Group (ECOG) 3590 study. Median survival was 57.5 months for LND group and $29.2 \%$ for LNS group $(\mathrm{p}=0.004) .(16)$

ACOSOG Z0030 trial was the largest prospective randomized trial comparing LNS and LND and it cannot be underestimated. Only $4 \%$ of additional patients were found to have positive lymph nodes at LND that were missed at LNS. This study clearly showed that LND does not improve long-term survival in patients with early-stage (T1 or T2, N0 or non-hilar N1) NSCLC who have pathologically negative lymph nodes after 
rigorous systematic LNS. However, interpretation of this study has to be made carefully because the rigorous systematic LNS performed in this study would not be realistic in a clinical practice in most of the centers and countries. Despite the similar survival between LNS group and LND group, the authors of this study still recommended that all patients with resectable NSCLC should undergo LND because the procedure provides the patients with the most accurate staging and the opportunity for effective adjuvant therapy without increasing mortality or morbidity. 
Hiroshi Date

\section{Figure legend}

\section{Figure 1}

These three figures demonstrate the differences in the surgical technique for lymph node assessment. A, lymph node biopsy. B, lymph node sampling. C, lymph node dissection. 
Figure 1

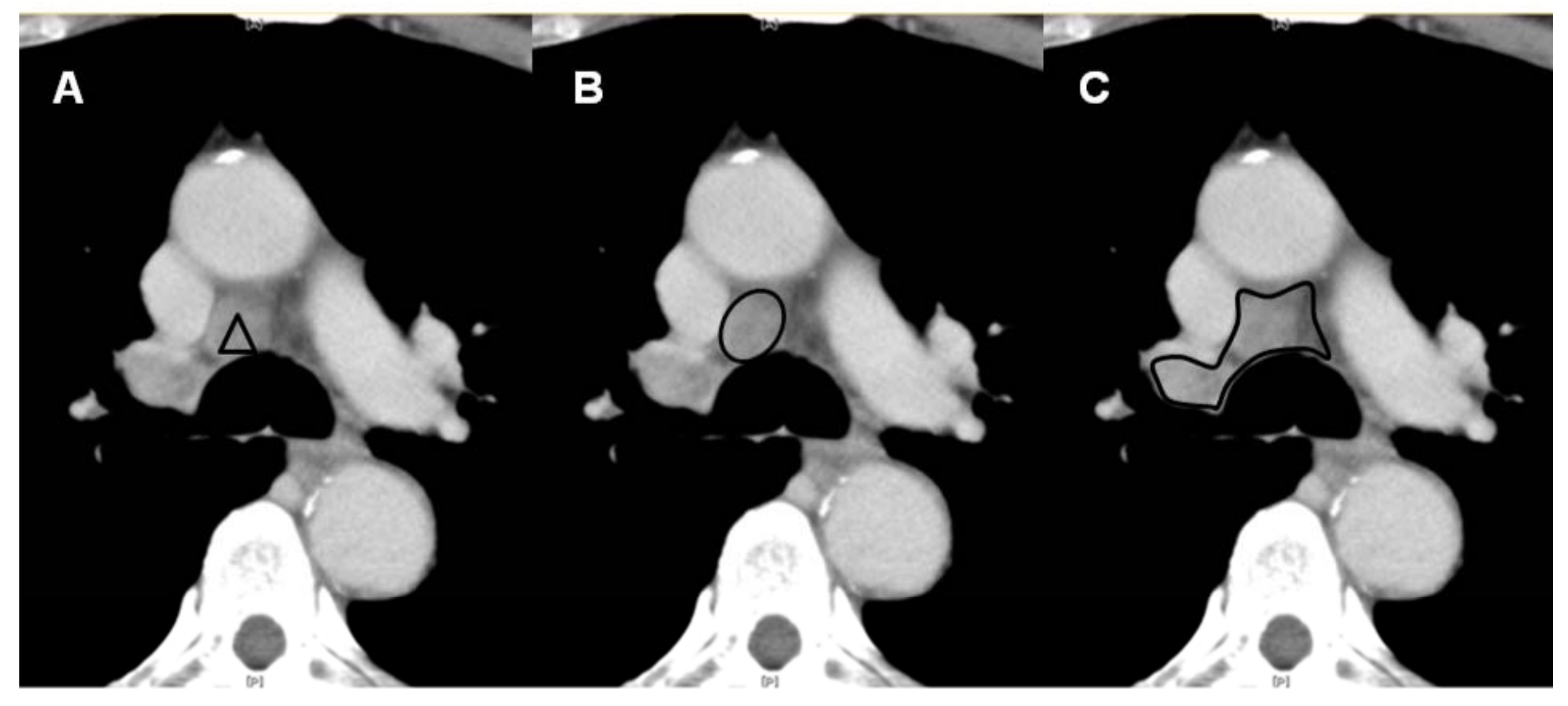




\section{REFERENCE LIST}

1. Mountain CF. Revisions in the International System for Staging Lung Cancer. Chest $1997 ; 111: 1710-7$.

2. Naruke T, Suemasu K, Ishikawa S. Lymph node mapping and curability at various levels of metastasis in resected lung cancer. J Thorac Cardiovasc Surg $1978 ; 76: 832-9$

3. Martini N, Flehinger BJ, Zaman MB, et al. Prospective study of 445 lung carcinomas with mediastinal lymph node metastases. J Thorac Cardiovasc Surg 1980;80:390-9.

4. Little AG, Rusch VW, Bonner JA, et al. Patterns of surgical care of lung cancer patients. Ann Thorac Surg 2005;80:2051-6.

5. Kato H, Ichinose Y, Ohata M, et al. A randomized trial of adjuvant chemotherapy with uracil-tegaful for adenocarcinoma of the lung. N Engl J Med 2004;350:1713-21.

6. Winton T, Livingston R, Johnson D, et al. Vinorelbine plus cisplatin vs observation in resected non-small-cell lung cancer. N Engl J Med 2005;352:2589-97.

7. Lardinois D, Leyn PD, Schil PV, et al. ESTS guidelines for intraoperative lymph node staging in non-small cell lung cancer. Euro $J$ Cardiothorac Surg 2006;30:787-92.

8. Asamura H. Lymph node dissection for lung cancer. In: General Thoracic Surgery. Philadelphia: Lippincott Williams \& Wilkins; 2009. p.1427-33.

9. Izbicki JR, Thetter $\mathrm{O}$, Habekost $\mathrm{M}$, et al. Radical systematic mediastinal lymphadenectomy in non-small cell lung cancer: a randomized controlled trial. $\mathrm{Br} \mathrm{J}$ Surg 1994;81:229-35.

10. Allen M, Darling GE, Pechet TTV et al. Morbidity and mortality of major pulmonary resections in patients with early-stage lung cancer: Initial results of the randomized, 
prospective ACOSOG Z0030 trial. Ann Thorac Surg 2006;81:1013-20.

11. Izbicki JR, Passlick B, Pantel K, et al. Effectiveness of radical systematic mediastinal lymphadenectomy in patients with resectable non-small cell lung cancer. Ann Surg 1998;227:138-44.

12. Sugi K, Nawata K, Fujita N, et al. Systematic lymph node dissection for clinically diagnosed peripheral non-small-cell lung cancer less than $2 \mathrm{~cm}$ in diameter. World $\mathrm{J}$ Surg 1998;22:290-5.

13. Wu Y, Huang Z, Wang S, et al. A randomized trial systematic nodal dissection in resectable non-small cell lung cancer. Lung Cancer 2002;36:1-6.

14. Darling GE, Allen MS, Decker PA, et al. Number of lymph nodes harvested from a mediastinal lymphadenectomy: Results of the randomized, prospective American College of Surgeons Oncology Group Z0030 trial. Chest 2011;139:1124-9.

15. Darling GE, Allen MS, Decker PA, et al. Randomized trial of mediastinal lymph node sampling versus complete lymph adrenectomy during pulmonary resection in the patient with N0 or N1 (less than hilar) non-small cell carcinoma: Results of the American College of Surgery Oncology Group Z0030 trial. J Thorac Cardiovasc Surg 2011;141:662-70.

16. Keller SM, Adak S. Wagner H, et al. Mediastinal lymph node dissection improves survival in patients with stage II and IIIa non-small cell lung cancer. Ann Thorac Surg 2000;70:358-66. 